\title{
Noise-induced transition from superfluid to vortex state in two-dimensional nonequilibrium polariton condensates
}

\author{
Vladimir N. Gladilin $\odot$ and Michiel Wouters \\ TQC, Universiteit Antwerpen, Universiteitsplein 1, B-2610 Antwerpen, Belgium
}

(Received 6 August 2019; revised manuscript received 18 November 2019; published 9 December 2019)

\begin{abstract}
We study the Berezinskii-Kosterlitz-Thouless mechanism for vortex-antivortex pair formation in twodimensional superfluids for nonequilibrium condensates. Our numerical study is based on a classical field model for driven-dissipative quantum fluids that is applicable to polariton condensates. We investigate the critical noise needed to create vortex-antivortex pairs in the systems, starting from a state with uniform phase. The dependence of the critical noise on the nonequilibrium and energy relaxation parameters is analyzed in detail.
\end{abstract}

DOI: 10.1103/PhysRevB.100.214506

\section{INTRODUCTION}

With the advent of synthetic quantum systems, the interest in driven-dissipative many-body systems has grown substantially in the last decade. Where particles in ultracold atomic gases can to a very good approximation be conserved, losses can be engineered [1] or are unavoidable in strongly interacting Bose gases [2]. In systems based on electromagnetic degrees of freedom, both in the microwave [3] and optical domain [4,5], cavity losses are often not negligible. In order to reach a steady state, some driving of the system is then necessary to compensate for the losses. This raises the question on the modifications of the steady state with respect to the thermal equilibrium state in conservative systems.

Here we will consider the case of two-dimensional weakly interacting bosons that are subject to single particle losses, which are compensated by a nonresonant drive. These systems are realized by microcavity polariton condensates [6], but there may be also the possibility to construct them with ultracold atoms [1]. Microcavity polaritons are hybrid light-matter quasiparticles resulting from the strong coupling between an excitonic transition in a quantum well and a photonic mode in an enveloping microcavity. From their photonic component, they inherit a light effective mass, enhancing the spatial coherence, whereas from their excitonic component, they inherit interactions (see Refs. [7,8] for the experimental determination of the interaction constant). Under nonresonant excitation, a large density of excitons is created, which subsequently relax to the lower polariton region. At equilibrium, the two-dimensional Bose gas features a Berezinskii-KosterlitzThouless (BKT) phase transition: for increasing temperature, thermally excited vortex-antivortex pairs become unbound, resulting in the loss of superfluidity. A natural question is then how this transition will be affected by losses and driving.

From the experimental side, this physics was addressed in Ref. [9]. In their system with a long polariton lifetime, they did find a phase transition that was interpreted as the binding to unbinding transition of vortex-antivortex pairs, reminiscent of the equilibrium situation.

Already at the level of small phase fluctuations, nonequilibrium systems behave differently from their equilibrium counterparts. Where for the latter, the phase dynamics is for small fluctuations to a good approximation described by a linear equation, in the nonequilibrium case, a nonlinear term appears, which brings them in the Kardar-Parisi-Zhang universality class [10-16].

Some first theoretical insight in the modification of the BKT transition due to the nonequilibrium condition can be gained by considering the modification of vortices when going away from equilibrium. It turns out that the gain/losses introduce an additional current with the vortex core as its source. The phase profile is consequently deformed, resulting in a spiral wave [17]. This modification in the vortex flow field subsequently affects the interactions between vortices and antivortices: when the outward flows are more important than the usual azimuthal flows, the interaction between vortex and antivortex becomes repulsive. These repulsive interactions hamper the vortex-antivortex recombination, enabling the formation of vortex-antivortex clusters with a very long lifetime [18]. A renormalization group based approach has shown that these repulsions are fatal for the superfluid phase. The renormalization flow always goes toward the normal phase, even though this physics may manifest itself only at very large distances [10].

In order to shed further light on the phase diagram of two-dimensional (2D) nonequilibrium polaritons, we resort here to numerical simulations of the noisy generalized GrossPitaevskii equation (ngGPE). This equation can be derived within the truncated Wigner approximation [19] or with the Keldysh field theory formalism [20,21] and has been widely used to model nonresonantly pumped polariton condensates [22-27]. At equilibrium, when the Bose gas is fully characterized by its density, temperature, and interaction constant, the critical temperature is found to equal $T_{c} \approx$ $2 \pi \hbar^{2} n /\left[m \ln \left(380 \hbar^{2} / m g\right)\right]$, where $n$ is the density, $m$ is the mass, and $g$ is the interaction strength [28]. Away from equilibrium however, there are more microscopic parameters that enter the theoretical description. We investigate here how they affect the critical noise strength (the analog of the temperature out of equilibrium).

In a previous theoretical study [18], based on a noise free generalized GPE, we found that starting from an initial state 
with a large number of vortices, several can survive in the steady state, because of the repulsive interactions between vortices and antivortices. We even found that these can form quite regular structures. With this physics understood, we will start here from the opposite initial condition with a homogeneous phase. In polariton condensates, such an initial condition can be achieved by sending a resonant pulse with a flat phase profile. As expected, we find that only when a sufficiently strong noise is present, vortex-antivortex pairs can be formed in the subsequent time evolution. We will show that the pair production shows a well defined noise threshold, allowing us to draw a phase diagram for the system.

For systems that are far from equilibrium, we have shown $[18,29]$ that the gGPE predicts a self-acceleration of vortices and production of new pairs in vortex-antivortex collisions, leading to chaotic dynamics. In this parameter regime we find that a moderate noise suppresses this mechanism, leading to a stabilization of the system.

The structure of the paper is as follows. In Sec. II our model for nonequilibrium condensation is recapitulated. The phase diagram is discussed in Sec. III and conclusions are drawn in Sec. IV.

\section{MODEL}

We consider nonresonantly excited two-dimensional polariton condensates. In the case of sufficiently fast relaxation in the exciton reservoir, this reservoir can be adiabatically eliminated and the condensate is described by the noisy generalized Gross-Pitaevskii equation [19-21,27,30,31]

$$
\begin{aligned}
(i-\kappa) \hbar \frac{\partial \psi}{\partial t}= & {\left[-\frac{\hbar^{2} \nabla^{2}}{2 m}+g|\psi|^{2}\right.} \\
& \left.+\frac{i}{2}\left(\frac{P}{1+|\psi|^{2} / n_{s}}-\gamma\right)\right] \psi+\sqrt{D} \xi .
\end{aligned}
$$

Here $m$ is the effective mass and the contact interaction between polaritons is characterized by the strength $g$. The imaginary term in the square brackets on the right-hand side describes the saturable pumping (with strength $P$ and saturation density $n_{s}$ ) that compensates for the losses $(\gamma)$. We take into account the energy relaxation $\kappa$ in the condensate [32,33]. The complex stochastic increments have the correlation function $\left\langle\xi^{*}(x, t) \xi\left(x^{\prime}, t^{\prime}\right)\right\rangle=2 \delta\left(\mathbf{r}-\mathbf{r}^{\prime}\right) \delta\left(t-t^{\prime}\right)$.

The dimensionless parameter $\kappa$ in the left-hand side of Eq. (1) describes energy relaxation and favors the formation of polariton condensates with lower energy to those with higher energy. Microscopic mechanisms that are responsible for energy relaxation are the scattering with phonons, but also scattering with the exciton reservoir and the energy dependence of the relaxation from the exciton reservoir to the lower polariton region $[34,35]$. In our model, the fluctuations that accompany this dissipation form part of the white noise. A more refined microscopic description of polariton-phonon scattering within the Gross-Pitaevskii equation was used in Refs. [36,37], but we restrict here to the simplest phenomenological model that is independent of the underlying microscopic mechanism.

For polariton condensates, the validity of Eq. (1) is not always straightforward to justify. The repulsive interactions between the condensate and the exciton reservoir may lead to an effective attraction between the polaritons, leading to instability for a positive polariton mass [24,38,39]. This unstable state can be stabilized by a negative effective mass that can be obtained in a polariton microcavity lattice [38]. We will not consider the instability physics in this work and assume positive mass and positive interactions [but the physics remains unaltered when the signs of the mass, interaction strength, and energy relaxation parameter $(\kappa)$ are simultaneously changed].

It is then convenient to rewrite Eq. (1) in a dimensionless form, by expressing the particle density $|\psi|^{2}$ in units of $n_{0} \equiv n_{s}(P / \gamma-1)$, time in units of $\hbar /\left(g n_{0}\right)$, length in units of $\hbar / \sqrt{2 m g n_{0}}$, and noise intensity in units of $\hbar^{3} n_{0} /(2 m)$ :

$$
\begin{aligned}
(i-\kappa) \frac{\partial \psi}{\partial t}= & {\left[-\nabla^{2}+|\psi|^{2}\right.} \\
& \left.+i c \frac{1-|\psi|^{2}}{1+\nu|\psi|^{2}}\right] \psi+\sqrt{D} \xi .
\end{aligned}
$$

Besides the noise intensity $D$, Eq. (2) contains three other dimensionless scalar parameters: $\kappa$ characterizes, as described above, damping in the system dynamics, $c=\gamma /\left(2 g n_{s}\right)$ is a measure of the deviation from equilibrium, and $v=n_{0} / n_{s}$ is proportional to the relative excess of the pumping intensity $P$ over the threshold intensity. In the absence of noise, Eq. (2) has the steady state solution $\psi=\sqrt{n} e^{-i n t}$, where the density $n$ satisfies

$$
\kappa n=\frac{c(1-n)}{1+v n},
$$

so that $n$ is a decreasing function of $\kappa$.

For weak noise, the fluctuations on top of this homogeneous solution can in first approximation be analyzed within the Bogoliubov approach [40], but a more refined analysis has revealed that the dynamics of the phase fluctuations is in the KPZ universality class [12-15,41,42], where the phase nonlinearity cannot be neglected.

Here we will study the critical noise needed to create vortices. In order to address this problem, Eq. (2) is solved numerically using the same finite-difference scheme as in Ref. [29]. Specifically, we use periodic boundary conditions for a square of size $L_{x}=L_{y}=40$ with grid step equal to 0.2 corresponding to a cutoff in momentum space equal to $K_{c}=5 \pi$. The model (1) is a classical field model that suffers from the usual ultraviolet catastrophe [28]. This implies that our results will be cutoff dependent. Below, we will discuss this dependence and how it will affect the comparison of our theoretical results with experiments.

When analyzing the noise-induced BKT transition, each run starts from a uniform condensate distribution at $D=$ 0 . Then we apply noise with a fixed nonzero intensity $D$ during a time interval $t_{D}$. The detection of vortex pairs in the presence of a strong noise is somewhat tricky, but fortunately in the absence of noise their annihilation time is known to be rather long even at very weak nonequilibrium $[23,43]$ and this time strongly increases with increasing $c$ [18]. For these reasons, it is more convenient to check the presence of vortex pairs sometime later after switching off the noise. The used time delay (typically few our units of time) is sufficient for 

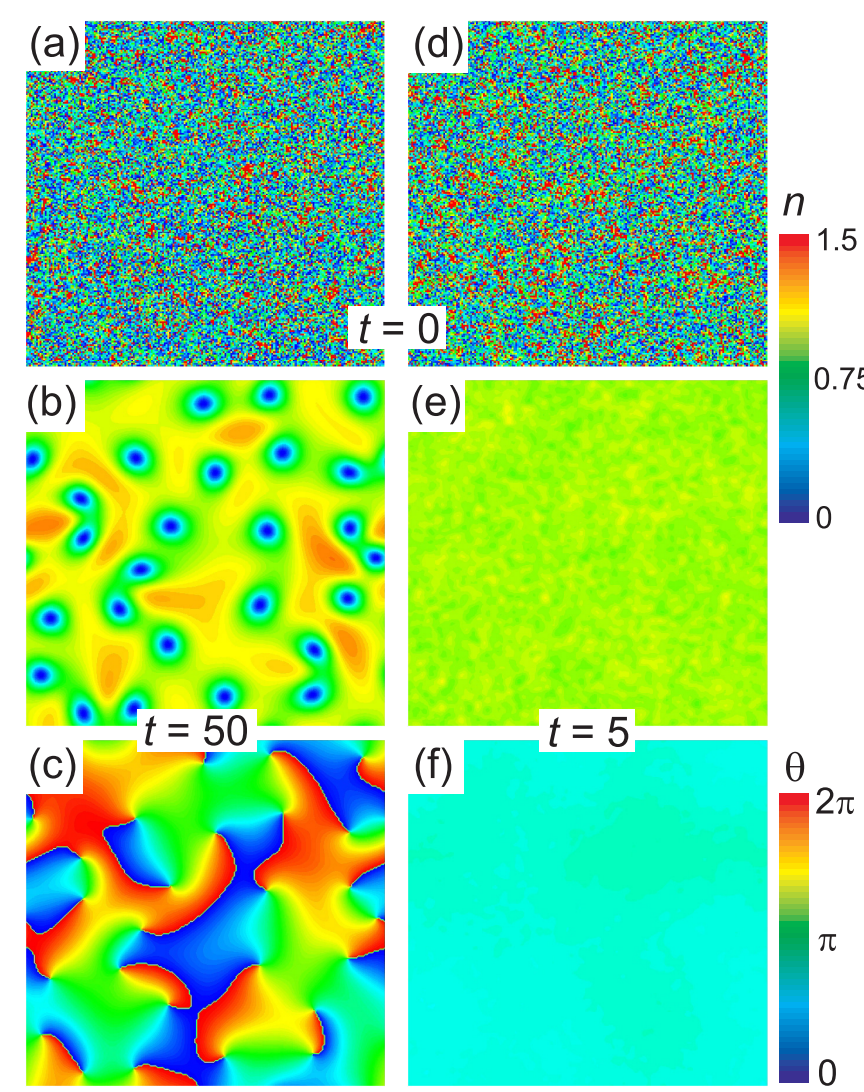

FIG. 1. Distributions of the particle density $n$ for $c=1.2, v=1$, $\kappa=0.01, D=0.015$, and $t_{D}=100$ at the time moment $t=0$ when the noise is switched off (a) and at $t=50$ (b). The distribution of the phase $\theta$ of the order parameter at $t=50$ is shown in (c). (d), (e), and (f) Same as (a), (b) and (c), respectively, but for $D=0.014, t_{D}=$ 600 , and a shorter time delay after switching off the noise $(t=5)$.

significant relaxation of the noisy component in the density and phase distributions of the condensate and, at the same time, is too short for vortex pair annihilation. To determine the critical noise for the BKT transition $D_{\mathrm{BKT}}$, we use the following criterion. If for a noise intensity $D$ vortex pairs are present after a noise exposure time $t_{D}$ (and hence $D>D_{\mathrm{BKT}}$ ), while for a certain noise intensity $D^{\prime}<D$ no vortex pairs appear even at noise exposures few times longer then $t_{D}$, then $D^{\prime}$ lies either below $D_{\mathrm{BKT}}$ or above $D_{\mathrm{BKT}}$ and closer to $D_{\mathrm{BKT}}$ then to $D$. Therefore, the critical noise intensity can be estimated as $D_{\text {BKT }}=D^{\prime} \pm\left(D-D^{\prime}\right)$. An illustration is displayed in Fig. 1. For $D=0.015$ and noise exposure time $t_{D}=100$, a noisy density distribution shown in Fig. 1(a) evolves after switching off the noise into a pattern with clearly seen vortices and antivortices, which persist during a relatively long time [see Figs. 1(b) and 1(c)]. The distance between these vortices and antivortices is substantially larger than the healing length, so that they should be considered as free (unbound). For a slightly lower noise intensity $D=0.014$, and significantly longer noise exposure time $t_{D}=600$, after switching off the noise the corresponding noisy distribution [Fig. 1(d)] rapidly relaxes towards a uniform vortex-free state [Figs. 1(e) and 1(f)]. According to our approach, the estimate for the critical noise in this case is $D_{\mathrm{BKT}}=0.014 \pm 0.001$.
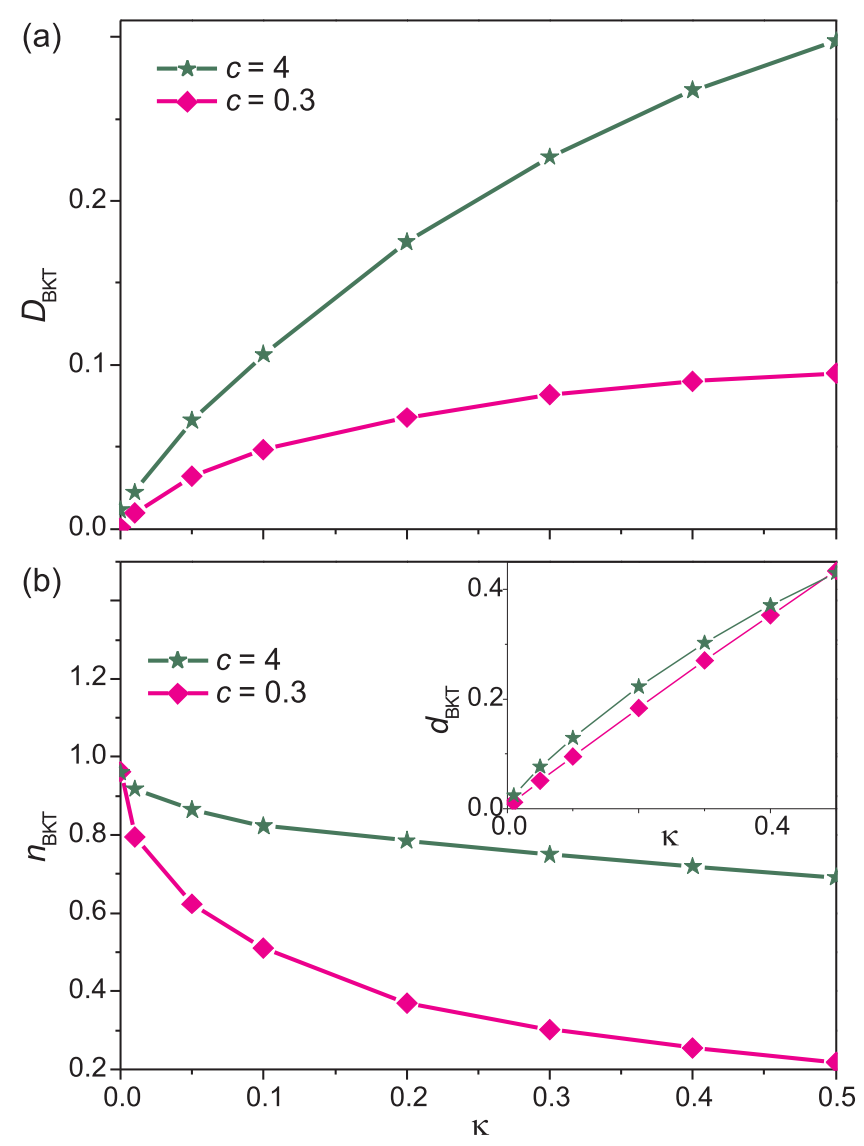

FIG. 2. Noise intensity $D_{\mathrm{BKT}}$ corresponding to the BKT transition (a) and average density of the condensate $n_{\mathrm{BKT}}$ at $D=D_{\mathrm{BKT}}$ (b) as a function of damping at $v=1$ and two different values of the nonequilibrium parameter $c$. The error bars are smaller than the symbol size. Inset of (b): Ratio $d_{\mathrm{BKT}}=D_{\mathrm{BKT}} / n_{\mathrm{BKT}}$ for $c=4$ (stars) and $c=0.3$ (diamonds).

\section{RESULTS AND DISCUSSION}

In Fig. 2(a) the critical noise intensity corresponding to the BKT transition $D_{\mathrm{BKT}}$ is shown as a function of the damping parameter $\kappa$ for the cases of moderate $(c=0.3)$ and strong $(c=4)$ deviations from equilibrium. In both cases the critical noise $D_{\mathrm{BKT}}$ is seen to considerably increase with $\kappa$, despite the fact that the average density of the condensate at $D=D_{\mathrm{BKT}}$ is a monotonously decreasing function of $\kappa$ [see also Eq. (3)], this decrease being especially pronounced at smaller $c$ [see Fig. 2(b)].

The inset of Fig. 2(b) shows the ratio $d_{\mathrm{BKT}} \equiv D_{\mathrm{BKT}} / n_{\mathrm{BKT}}$ as a function of $\kappa$. For the two very different values of the nonequilibrium parameter $c$, the curves $d_{\mathrm{BKT}}(\kappa)$ appear to lie relatively close to each other. One can also notice that at $c=$ 0.3 the dependence of $d_{\mathrm{BKT}}$ on $\kappa$ is nearly linear (except for the smallest values of $\kappa$ ).

At $\kappa \gg 1$ linear scaling of the noise intensity with $\kappa$ directly follows from an additional rescaling of time $[t \rightarrow$ $t / \kappa$; see Eq. (2)]. Our numerical results show that this linear dependence of $d_{\mathrm{BKT}}$ on $\kappa$ remains to good approximation unaltered even when $\kappa<1$.

At equilibrium $(c=0)$, the increase of the critical noise with increasing $\kappa$ can be understood by making the connection 
with the thermal equilibrium case. For vanishing nonequilibrium and $\kappa \gg 1$, our equation reduces to model A dynamics [44], which has a Boltzmann-Gibbs steady state distribution at temperature $T=D / 2 \kappa$. In this limit, the transition occurs at the equilibrium BKT temperature [28], where $T_{\mathrm{BKT}}=\eta n$, with $\eta$ a numerical cutoff dependent constant. For the critical noise, one then obtains $D_{\mathrm{BKT}}=2 \eta \kappa n$.

Numerically, we have observed that for $c \rightarrow 0$, the critical noise obeys this relation, even for $\kappa<1$. The fact that the dissipative part of the dynamics does not alter the steady state can be understood from the following argument. It is well established that pure Gross-Pitaevskii dynamics $(D=$ $\kappa=c=0$ ) samples the thermal equilibrium state in the microcanonical ensemble, at an energy determined by the initial state. Similarly, the Langevin dynamics [when omitting the $i$ in the left-hand side of Eq. (2) and taking $c=0$ ] samples the phase space according to the canonical ensemble at a temperature determined by the balance between noise and dissipation. Since the thermal state is the steady state of both the GP and Langevin dynamics, it is natural that the steady state of the combined dynamics is also at thermal equilibrium, with the temperature determined by the Langevin part.

As implied by the results displayed in Fig. 2(a), the critical noise intensity $D_{\text {BKT }}$ increases when moving away from equilibrium. The dependence of $D_{\mathrm{BKT}}$ on the nonequilibrium parameter $c$ is further illustrated in Fig. 3(a) for the cases of weak $(\kappa=0.01$ and 0.1$)$ and zero damping. At nonzero damping the increase of $D_{\mathrm{BKT}}$ with $c$ is partly related to the simultaneous increase of the average condensate density $n_{\mathrm{BKT}}(c)$ shown in Fig. 3(b). The latter originates from the growing contribution of the pumping-loss term in Eq. (2) [the last term in the square brackets in Eq. (2)]. Indeed, this term tends to keep the condensate density as close to 1 as possible. As a result, the degrading effect of damping or noise on the condensate density weakens with increasing $c$. This can be seen, e.g., from Fig. 2(b) by comparing to each other the values of $n_{\mathrm{BKT}}$ at different $c$ : for each fixed, not too small $\kappa$, the value of $\left.n_{\mathrm{BKT}}\right|_{c=4}$ is significantly larger than $\left.n_{\mathrm{BKT}}\right|_{c=0.3}$, even though the density $\left.n_{\mathrm{BKT}}\right|_{c=4}$ corresponds to a considerably higher noise intensity than that for $\left.n_{\mathrm{BKT}}\right|_{c=0.3}$ [see Fig. 2(a)].

At the same time, as follows from the behavior of the ratio $d_{\mathrm{BKT}} \equiv D_{\mathrm{BKT}} / n_{\mathrm{BKT}}$ [see the inset of Fig. 3(a)], the increase of $D_{\mathrm{BKT}}$ with $c$ is considerably faster as compared to that of $n_{\mathrm{BKT}}$. This means that the effect of pumping-loss processes on $D_{\mathrm{BKT}}$ cannot be explained solely by the corresponding stabilization of the average condensate density against noise and damping. This becomes even more evident when looking at the results for $\kappa=0$. Indeed, while the critical noise $D_{\mathrm{BKT}}$ demonstrates a clear rise with increasing $c$ [see Fig. 3(a)], the average density $n_{\mathrm{BKT}}(c)$ remains almost constant [see Fig. 3(b)]. At small $c$, the values of $n_{\mathrm{BKT}}$ even slightly decrease with $c$ suggesting that the stabilizing effects of the pumping-loss processes do not completely compensate the average-density reduction caused by the increase of the noise intensity at the BKT transition. However, an important aspect of the the pumping-loss processes, which is not directly reflected in the average density, is that they impede formation of deep local suppressions of the condensate density. Those deep density suppressions, together with the appropriate phase gradient, are
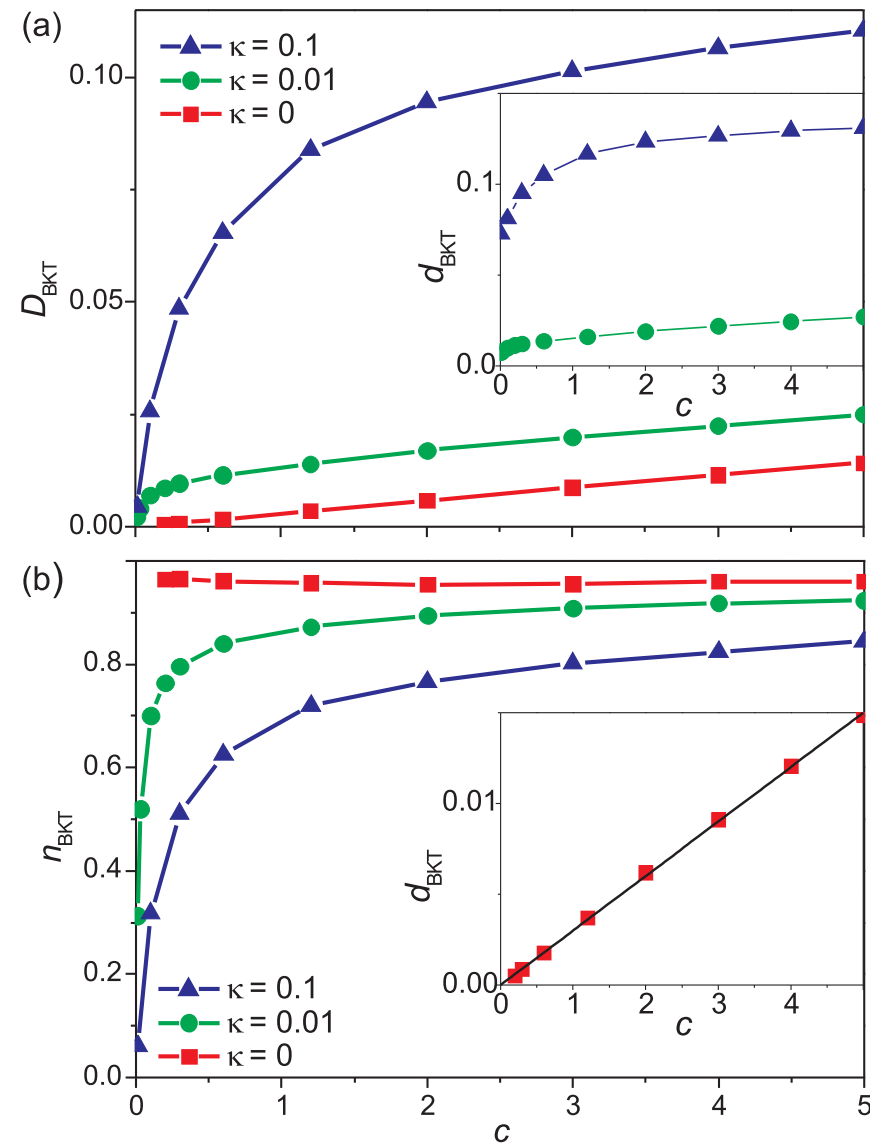

FIG. 3. Noise intensity $D_{\mathrm{BKT}}$ corresponding to the BKT transition (a) and average density of the condensate $n_{\mathrm{BKT}}$ at $D=D_{\mathrm{BKT}}$ (b) as a function of the nonequilibrium parameter $c$ at $v=1$ and different values of the damping parameter $\kappa$. The error bars are smaller than the symbol size. Inset of (a): Ratio $d_{\mathrm{BKT}}=D_{\mathrm{BKT}} / n_{\mathrm{BKT}}$ for $\kappa=0.1$ (triangles) and $\kappa=0.01$ (circles). Inset of (b): Ratio $d_{\mathrm{BKT}}=D_{\mathrm{BKT}} / n_{\mathrm{BKT}}$ for $\kappa=0$ (squares). The black line corresponds to the linear fitting $d_{\mathrm{BKT}}=a c$, with $a=0.003$.

necessary prerequisites for vortex pair formation and hence for the BKT transition. The discussed numerical results imply that just the stabilizing effect of the pumping-loss term on the local density of the condensate governs the increase of $D_{\text {BKT }}$ with $c$ at $\kappa \rightarrow 0$. At $\kappa=0$ the dependence of $D_{\text {BKT }}$ on $c$ becomes close to linear [see Fig. 3(a)], while for $d_{\mathrm{BKT}}(c)$ a linear function $d=a c$ with $a=0.003$ provides a nearly perfect approximation [see Fig. 3(b)].

This interpretation of our numerical results can be elucidated by a linear analysis of the fluctuations. Writing the field as $\psi(x, t)=\sqrt{1+\delta n(x, t)} e^{i \theta(x, t)}$, one obtains in linearized approximation for the Fourier components

$$
\begin{aligned}
\frac{\partial}{\partial t}\left(\begin{array}{c}
\theta_{k} \\
n_{k}
\end{array}\right)= & \left(\begin{array}{cc}
-\kappa \epsilon_{k} & -\frac{\epsilon_{k}}{2}-1 \\
2 \epsilon_{k} & -\frac{2 c}{1+\nu}+4 \kappa
\end{array}\right)\left(\begin{array}{c}
\theta_{k} \\
n_{k}
\end{array}\right) \\
& +\left(\begin{array}{c}
\sqrt{D} \xi_{k}^{(\theta)} \\
\sqrt{4 D} \xi_{k}^{(n)}
\end{array}\right),
\end{aligned}
$$

where $\epsilon_{k}=k^{2}$. The white noise in Fourier space has the correlation function $\left\langle\xi^{(\theta)}(k, t) \xi^{(\theta)}\left(k^{\prime}, t\right)\right\rangle=4 \pi \delta\left(k+k^{\prime}\right) \delta(t-$ $\left.t^{\prime}\right)$, analogous for $\xi^{(n)}$ and $\left\langle\xi^{(\theta)} \xi^{(n)}\right\rangle=0$. 


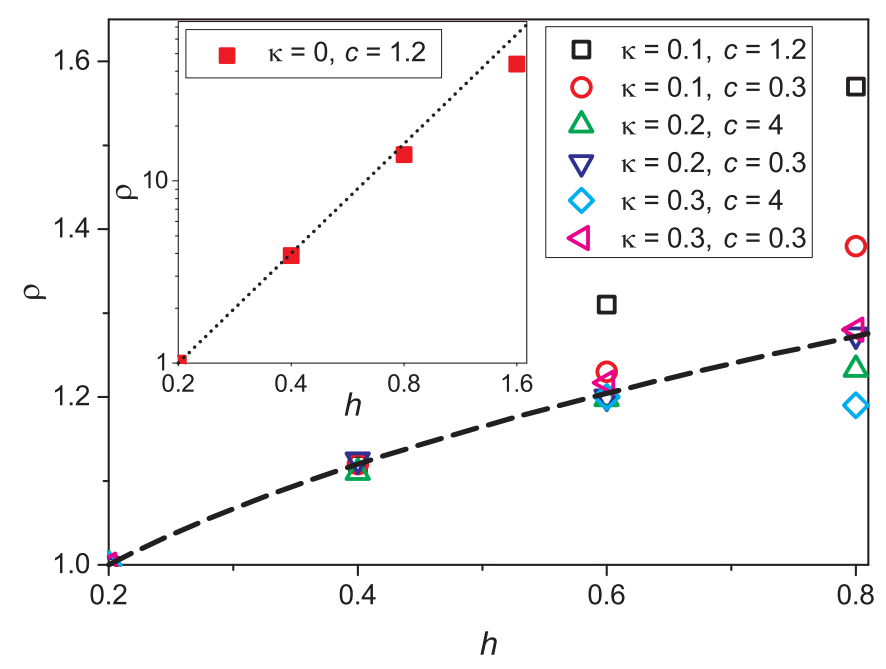

FIG. 4. Ratio $\rho \equiv d_{\mathrm{BKT}}(\kappa, c, h) / d_{\mathrm{BKT}}(\kappa, c, h=0.2)$ as a function of the grid step $h$ for $v=1$ and different values of $\kappa$ and $c$. The dashed line corresponds to the dependence $\rho=\ln (b / 0.2) / \ln (b / h)$ with $b=130$. The error bars are smaller than the symbol size. Inset: Ratio $\rho(h)$ for $v=1, \kappa=0$, and $c=1.2$. The dotted line corresponds to the dependence $\rho(h)=(h / 0.2)^{2}$.

The steady state density fluctuations can be obtained in closed form. In the limit of large $k$, they simplify to .

$$
\left\langle n_{k} n_{k^{\prime}}\right\rangle= \begin{cases}2 \pi \delta\left(k+k^{\prime}\right) \frac{4 D\left(2+\kappa^{2}\right)}{\kappa \epsilon_{k}}, & \kappa \neq 0, \\ 2 \pi \delta\left(k+k^{\prime}\right) \frac{8 D(1+\nu)}{c}, & \kappa=0 .\end{cases}
$$

For the density fluctuations in real space, we then obtain for a sufficiently large momentum cutoff $K_{c}$,

$$
\left\langle\delta n^{2}(x)\right\rangle \propto \begin{cases}\frac{D}{\kappa}\left(2+\kappa^{2}\right) \ln K_{c}, & \kappa \neq 0, \\ \frac{D(1+v)}{c} K_{c}^{2}, & \kappa=0 .\end{cases}
$$

The local density fluctuations obtained here in the linear approximation show behavior that is in line with what was observed numerically for the BKT transition. Density fluctuations are first suppressed by the damping $\kappa$ and for $\kappa=0$ by the nonequilibrium parameter $c$.

Note the very different dependence of both results on the momentum cutoff. In the presence of a nonzero damping, the cutoff dependence is a very weak logarithm, where in its absence, it becomes quadratic. This difference is due to the fact that the momentum space density tends to a constant in the $\kappa=0$ case, where it decays as $k^{-2}$ for $\kappa \neq 0$. Even the latter is not fast enough to ensure convergence in two dimensions, hence the logarithmic divergence of the density fluctuations.

The numerically observed dependence of $D_{\mathrm{BKT}}$ on the grid spacing correlates with the above analysis, as illustrated in Fig. 4. The symbols show the ratio $\rho \equiv$ $d_{\mathrm{BKT}}(\kappa, c, h) / d_{\mathrm{BKT}}(\kappa, c, h=0.2)$ at different values of the grid step $h$ for $\nu=1$ and different $\kappa$ and $c$. The dashed line shows a logarithmic $h$ dependence inspired by Eq. (7): $\rho=\ln (b / 0.2) / \ln (b / h)$ with $b=130$. It is seen to fit well the behavior of the numerical results at smaller $h$. In the inset of Fig. 4, we show the dependence of $\rho$ on the grid step for $\kappa=0$, together with a quadratic fit [see Eq. (8)], which is very good except for the largest grid spacing. The logarithmic cutoff dependence for $\kappa>0$ is implied by Eq. (7) when assuming that the density fluctuations should lead to a full local density suppression as a prerequisite of vortexpair formation. At larger $h$ and the relatively small value $\kappa=0.1$, the increase of the numerically determined $\rho$ with $h$ is clearly faster than that predicted by Eq. (7). This can be considered as a reminiscence of the quadratic dependence of the density fluctuations on $K_{c}$, as derived in the limit $\kappa \rightarrow 0$ [see Eq. (8)]. When $h$ approaches 1 , also for large $\kappa$, discrepancies appear. This is likely due to the fact that at those grid spacings, the shape of a vortex core is not correctly described in the simulations (recall that our size units is determined by the healing length of the condensate). Consequently, the formation of a vortex pair does not require a density suppression (at $h \gg 1$, no density suppression may occur). As result, the critical noise level is overestimated by our logarithmic estimate. As implied by Fig. 4, the difference is more pronounced at strong deviations from equilibrium (see the results for $h=0.8, \kappa=0.2,0.3$, and $c=4$ ). At large grid steps, a downward deviation of the numerically obtained $\rho(h)$ from the analytically estimated quadratic dependence is seen also for the case of $\kappa=0$ in the inset of Fig. 4 .

As seen from Eq. (2), the pumping-loss term increases in magnitude when decreasing the parameter $v$. One can expect, therefore, that a decrease of $v$ leads to an increase of the critical noise $D_{\text {ВKT }}$. Our simulations confirm this expectation but, at the same time, show that the influence of $v$ on $D_{\text {BKT }}$ is relatively weak for nonvanishing damping. At $\kappa=0.01$ a decrease of $v$ by one order of magnitude (from 1 down to 0.1 ) results in an increase of $D_{\mathrm{BKT}}$ approximately by $4 \%$ at $c=0.3$ and by $12 \%$ at $c=4$. The corresponding increase of $d_{\mathrm{BKT}}$ is about $20 \%$ for both $c=0.3$ and 4 . In other words, the effect of $v$ on the critical noise is rather minor as compared to the much stronger impact of $\kappa$ and $c$.

Let us now look in some more detail at the simultaneous dependence of $d_{\mathrm{BKT}}$ on $\kappa$ and $c$. Taking into account, on the one hand, the nearly linear dependence $d_{\mathrm{BKT}}$ on $\kappa$ at large $\kappa$ [see the inset in Fig. 2(b)] and, on the other hand, the linear dependence on $c, d_{\mathrm{BKT}} \approx a c$ at $\kappa \rightarrow 0$, it seems reasonable to consider the renormalized quantity $d_{\mathrm{BKT}} /(\kappa+$ $a c)$. As seen from Fig. 5, all the results of our simulations lie in a rather narrow interval around $1: 0.6<d_{\mathrm{BKT}} /(\kappa+$ $a c)<1.3$. This suggests that the simple expression $\kappa+$ $0.003 c$ already can serve as a crude but quite reasonable estimate for $d_{\mathrm{BKT}}$ at any $\kappa$ and $c$. A better approximation of the numerical results is obtained (see Fig. 5) by using the function $d^{*}=0.609 \kappa+0.003 c+\kappa^{1.411} c^{0.411} /\left(2.646 \kappa^{0.610}+\right.$ $\left.0.0706 c^{0.889}+1.622 \kappa^{1.514} c^{1.308}\right)$, constructed as a result of a purely mathematical fitting. Since the numerical results for the dependence of $d_{\mathrm{BKT}}$ on both $\kappa$ and $c$ are seen to be described quite well by $d^{*}(c, \kappa)$, we may expect that this function can provide also meaningful results for inter- and extrapolation within the system under consideration [see the inset in Fig. 5(b)].

Finally, we address the question of whether the BKT transition under consideration can be influenced by the effect of vortex pair generation by moving vortices [18]. This effect has been predicted to occur in strongly nonequilibrium condensates when self-accelerated vortices acquire sufficiently 

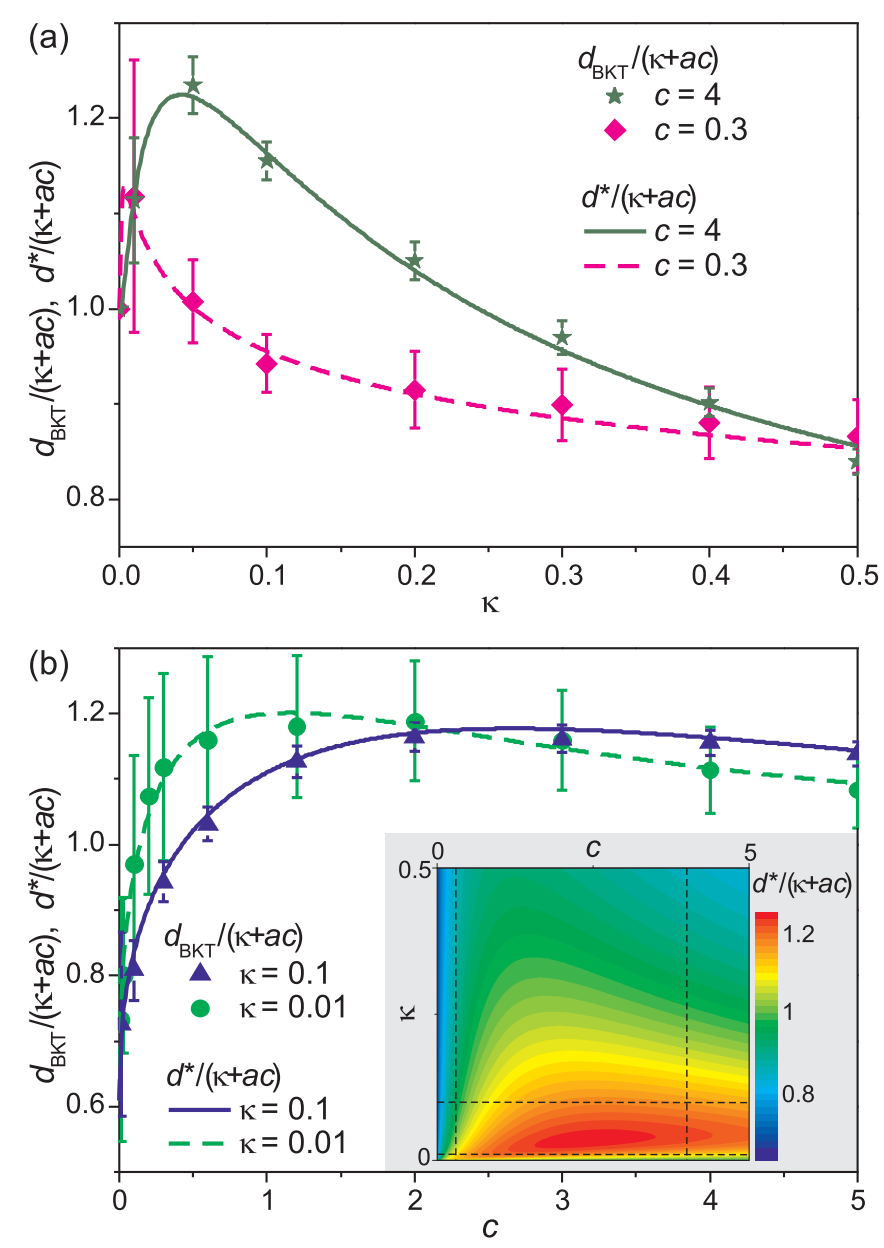

FIG. 5. Renormalized noise intensity $d_{\mathrm{BKT}} /(\kappa+a c)$ (symbols) and its fitting by $d^{*} /(\kappa+a c)$ (lines) as a function of the damping parameter $\kappa$ (a) and nonequilibrium parameter $c$ (b). The inset shows the dependence of $d^{*} /(\kappa+a c)$ on both $c$ and $\kappa$. Straight dashed lines correspond to the parameter values covered by the curves in (a) and (b).

high velocities with respect to the surrounding condensate. The results of [18] were obtained in the absence of noise. In our numerics containing the noise term, we have observed that fluctuations of the condensate density and currents tend to impede the acceleration of vortices. Consequently, the generation of vortex pairs in vortex collisions becomes impossible at high noise intensities. This is illustrated in Fig. 6, which shows the upper boundary $D_{\mathrm{m}}$ for the noise intensity range, where generation of new vortex pairs by self-accelerated vortices is possible, as a function of $c$ at $\nu=1$ and $\kappa=0.01$. The corresponding calculations are performed, like in [18], starting with one pinned vortex pair in the region under consideration and simulating the dynamics during a time interval $\Delta t \sim 1000$ after depinning of vortices, now in the presence of noise.

In Fig. 6 the dependence of $D_{\mathrm{m}}$ on $c$ is given in comparison with the critical noise $D_{\mathrm{BKT}}$ at the same $\kappa$ and $v$. As seen from Fig. 6 , the region of $c$ and $D$, where pair generation by vortices is possible, lies at large $c(c>3.3)$ and has no overlap with the curve $D_{\mathrm{BKT}}(c)$. Of course the latter remains true for any $\kappa \geqslant 0.01$. Indeed, the critical noise $D_{\mathrm{BKT}}$ increases with $\kappa$. At the same time, damping slows down vortex motion and thus impedes pair generation by moving vortices. As a

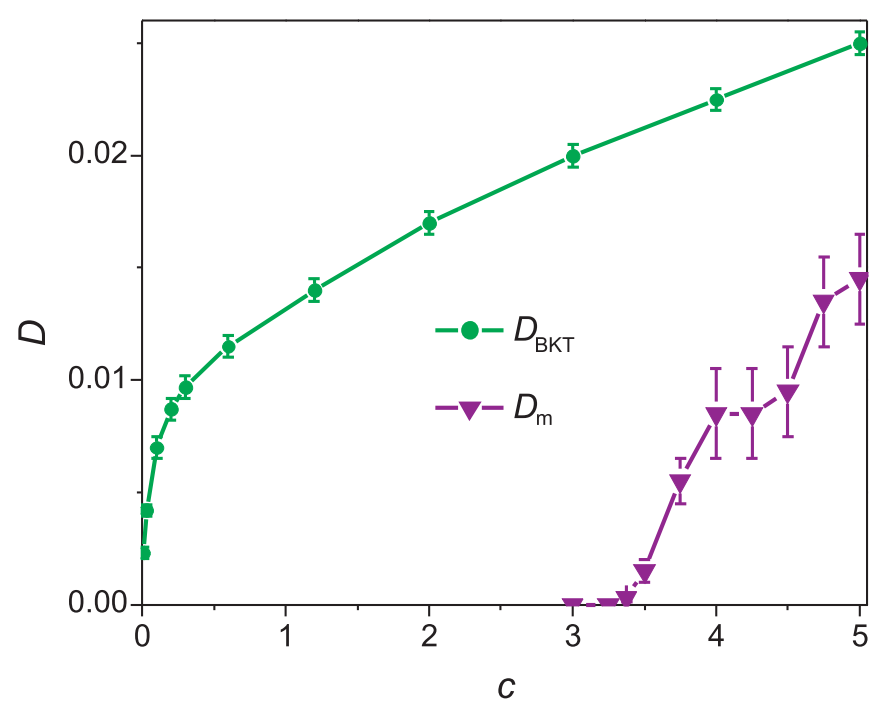

FIG. 6. Noise intensity $D_{\mathrm{BKT}}(c)$ corresponding to the BKT transition in comparison with $D_{\mathrm{m}}(c)$, the maximum noise intensity, which still allows for vortex pair generation by moving vortices, at $v=1$ and $\kappa=0.01$.

result, the aforementioned region will shrink with increasing $\kappa$. Our simulations show that already at $\kappa$ as small as 0.1 the velocities of vortices are insufficient for pair generation. So we can conclude that at $\kappa \geqslant 0.01$ the BKT transition is not affected by the processes of pair generation by moving vortices. As concerns the limit of very weak damping $\kappa<$ 0.01 , the nearly linear behavior of $D_{\mathrm{BKT}}(c)$, obtained for $\kappa=0$, manifests no visible peculiarities in the region of large $c$, where the processes of pair generation by vortices become possible. This implies that, also in the limit $\kappa \rightarrow 0$, the BKT transition is not considerably affected by these processes.

\section{CONCLUSIONS}

We have investigated the critical noise for the spontaneous formation of unbound vortex-antivortex pairs in a drivendissipative bosonic system where particle losses are compensated by nonresonant pumping. In this work we have focused on the noise needed to form vortex-antivortex pairs starting from a uniform phase. In our model, the critical noise strength depends - with a suitable choice of units-on three dimensionless parameters: the energy relaxation rate $(\kappa)$, the nonequilibrium parameter $(c)$, and the gain saturation parameter $(v)$.

In the absence of energy relaxation, the nonequilibrium parameter determines the critical noise strength, but this critical value depends quadratically on the momentum space cutoff, giving our numerical results limited predictivity for specific experiments. In the presence of sufficiently strong energy relaxation, the cutoff dependence is much weaker and experimentally relevant results can be extracted from the numerics in this case. In this regime, the critical noise strength increases both with energy relaxation (as expected from equilibrium calculations) and with nonequilibrium parameter $c$. The latter dependence could seem counterintuitive, because with increasing $c$, vortices and antivortices repel each other at large distances, which could favor their unbinding. We 
interpret the impeding of vortex-antivortex formation further away from nonequilibrium as a consequence of the reduction of the density fluctuations. The effect of nonequilibrium on the formation of vortices is therefore opposite to its effect on the annihilation: the lifetime of existing vortices is dramatically enhanced by nonequilibrium [18].

Quantitatively, we have found that the effect of the nonequilibrium parameter is actually small for $\kappa \gg 0.003 c$, which is satisfied when damping is not too small and the system is not too far from equilibrium. Very far from equilibrium, self-acceleration of vortices can lead to the production of new vortex antivortex pairs. We have shown here that this pair production ceases for increasing noise.
Our numerical simulations were performed for systems with periodic boundary conditions whose size is comparable to the systems employed in current experiments. The study of the thermodynamical limit of infinite system size remains a challenge for both theoretical analysis and experimental investigation.

\section{ACKNOWLEDGMENTS}

We thank Sebastian Diehl for a stimulating discussion. This work was financially supported by the Flemish Science Foundation (FWO) Odysseus program and the Research Council of Antwerp University (UA-BOF) project 150168.
[1] R. Labouvie, B. Santra, S. Heun, and H. Ott, Phys. Rev. Lett. 116, 235302 (2016).

[2] P. Makotyn, C. E. Klauss, D. L. Goldberger, E. A. Cornell, and D. S. Jin, Nat. Phys. 10, 116 (2014); C. Eigen, J. A. P. Glidden, R. Lopes, E. A. Cornell, R. P. Smith, and Z. Hadzibabic, Nature (London) 563, 221 (2018).

[3] M. Fitzpatrick, N. M. Sundaresan, A. C. Y. Li, J. Koch, and A. A. Houck, Phys. Rev. X 7, 011016 (2017).

[4] I. Carusotto and C. Ciuti, Rev. Mod. Phys. 85, 299 (2013).

[5] F. Brennecke, R. Mottl, K. Baumann, R. Landig, T. Donner, and T. Esslinger, Proc. Natl. Acad. Sci. 110, 11763 (2013).

[6] J. Kasprzak, M. Richard, S. Kundermann, A. Baas, P. Jeambrun, J. Keeling, F. Marchetti, M. Szymańska, R. Andre, J. Staehli, V. Savona, P. B. Littlewood, B. Deveaud, and L. S. Dang, Nature (London) 443, 409 (2006).

[7] A. Delteil, T. Fink, A. Schade, S. Höfling, C. Schneider, and A. Imamoğlu, Nat. Mater. 18, 219 (2019).

[8] S. R. K. Rodriguez, W. Casteels, F. Storme, N. Carlon Zambon, I. Sagnes, L. Le Gratiet, E. Galopin, A. Lemaître, A. Amo, C. Ciuti, and J. Bloch, Phys. Rev. Lett. 118, 247402 (2017).

[9] D. Caputo, D. Ballarini, G. Dagvadorj, C. S. Muñoz, M. De Giorgi, L. Dominici, K. West, L. N. Pfeiffer, G. Gigli, F. P. Laussy, M. H. Szymańska, and D. Sanvitto, Nat. Mater. 17, 145 (2018).

[10] G. Wachtel, L. M. Sieberer, S. Diehl, and E. Altman, Phys. Rev. B 94, 104520 (2016).

[11] L. M. Sieberer, G. Wachtel, E. Altman, and S. Diehl, Phys. Rev. B 94, 104521 (2016).

[12] D. Squizzato, L. Canet, and A. Minguzzi, Phys. Rev. B 97, 195453 (2018).

[13] V. N. Gladilin, K. Ji, and M. Wouters, Phys. Rev. A 90, 023615 (2014).

[14] L. He, L. M. Sieberer, E. Altman, and S. Diehl, Phys. Rev. B 92, 155307 (2015).

[15] E. Altman, L. M. Sieberer, L. Chen, S. Diehl, and J. Toner, Phys. Rev. X 5, 011017 (2015).

[16] L. M. Sieberer, M. Buchhold, and S. Diehl, Rep. Progress Phys. 79, 096001 (2016).

[17] I. S. Aranson and L. Kramer, Rev. Mod. Phys. 74, 99 (2002).

[18] V. N. Gladilin and M. Wouters, J. Phys. A: Math. Theor. 52, 395303 (2019).

[19] M. Wouters and V. Savona, Phys. Rev. B 79, 165302 (2009).
[20] M. H. Szymanśka, J. Keeling, and P. B. Littlewood, Phys. Rev. B 75, 195331 (2007).

[21] L. M. Sieberer, S. D. Huber, E. Altman, and S. Diehl, Phys. Rev. B 89, 134310 (2014).

[22] K. P. Kalinin, P. G. Lagoudakis, and N. G. Berloff, Phys. Rev. B 97, 094512 (2018).

[23] P. Comaron, G. Dagvadorj, A. Zamora, I. Carusotto, N. P. Proukakis, and M. H. Szymańska, Phys. Rev. Lett. 121, 095302 (2018).

[24] N. Bobrovska, M. Matuszewski, K. S. Daskalakis, S. A. Maier, and S. Kéna-Cohen, ACS Photon. 5, 111 (2017).

[25] E. Estrecho, T. Gao, N. Bobrovska, M. D. Fraser, M. Steger, L. Pfeiffer, K. West, T. C. H. Liew, M. Matuszewski, D. W. Snoke, A. G. Truscott, and E. A. Ostrovskaya, Nat. Commun. 9, 2944 (2018).

[26] H. Ohadi, Y. del Valle-Inclan Redondo, A. J. Ramsay, Z. Hatzopoulos, T. C. H. Liew, P. R. Eastham, P. G. Savvidis, and J. J. Baumberg, Phys. Rev. B 97, 195109 (2018).

[27] J. Keeling and N. G. Berloff, Phys. Rev. Lett. 100, 250401 (2008).

[28] N. Prokof'ev, O. Ruebenacker, and B. Svistunov, Phys. Rev. Lett. 87, 270402 (2001).

[29] V. N. Gladilin and M. Wouters, New J. Phys. 19, 105005 (2017).

[30] M. Wouters and I. Carusotto, Phys. Rev. Lett. 99, 140402 (2007).

[31] N. Bobrovska and M. Matuszewski, Phys. Rev. B 92, 035311 (2015).

[32] L. P. Pitaevskii, Zh. Eksp. Teor. Fiz. 35, 408 (1958) [Sov. Phys. JETP 35, 282 (1959)].

[33] M. Wouters, New J. Phys. 14, 075020 (2012).

[34] F. Tassone, C. Piermarocchi, V. Savona, A. Quattropani, and P. Schwendimann, Phys. Rev. B 56, 7554 (1997).

[35] D. Porras, C. Ciuti, J. J. Baumberg, and C. Tejedor, Phys. Rev. B 66, 085304 (2002).

[36] I. G. Savenko, T. C. H. Liew, and I. A. Shelykh, Phys. Rev. Lett. 110, 127402 (2013).

[37] S. Klembt, P. Stepanov, T. Klein, A. Minguzzi, and M. Richard, Phys. Rev. Lett. 120, 035301 (2018).

[38] F. Baboux, D. De Bernardis, V. Goblot, V. N. Gladilin, C. Gomez, E. Galopin, L. Le Gratiet, A. Lemaître, I. Sagnes, I. Carusotto, M. Wouters, A. Amo, and J. Bloch, Optica 5, 1163 (2018). 
[39] N. Bobrovska, E. A. Ostrovskaya, and M. Matuszewski, Phys. Rev. B 90, 205304 (2014).

[40] A. Chiocchetta and I. Carusotto, Europhys. Lett. 102, 67007 (2013).

[41] K. Ji, V. N. Gladilin, and M. Wouters, Phys. Rev. B 91, 045301 (2015).
[42] L. He and S. Diehl, New J. Phys. 19, 115012 (2017).

[43] M. Kulczykowski and M. Matuszewski, Phys. Rev. B 95, 075306 (2017).

[44] P. C. Hohenberg and B. I. Halperin, Rev. Mod. Phys. 49, 435 (1977). 